\title{
Endpoint Estimates for Fractional Hardy Operators and Their Commutators on Hardy Spaces
}

\author{
Jiang Zhou and Dinghuai Wang \\ Department of Mathematics, Xinjiang University, Urumqi 830046, China \\ Correspondence should be addressed to Jiang Zhou; zhoujiangshuxue@126.com
}

Received 19 October 2013; Revised 28 December 2013; Accepted 30 December 2013; Published 18 February 2014

Academic Editor: Yongqiang Fu

Copyright (C) 2014 J. Zhou and D. Wang. This is an open access article distributed under the Creative Commons Attribution License, which permits unrestricted use, distribution, and reproduction in any medium, provided the original work is properly cited.

$\left(H^{p}\left(\mathbb{R}^{n}\right), L^{q}\left(\mathbb{R}^{n}\right)\right)$ bounds of fractional Hardy operators are obtained. Moreover, the estimates for commutators of fractional Hardy operators on Hardy spaces are worked out. It is also proved that the commutators of fractional Hardy operators are mapped from the Herz-type Hardy spaces into the Herz spaces. The estimates for multilinear commutators of fractional Hardy operators are also discussed.

\section{Introduction}

The most fundamental averaging operator is the Hardy operators defined by

$$
H(f)(x)=\frac{1}{x} \int_{0}^{x} f(t) d t
$$

where the function $f$ is nonnegative integrable on $\mathbb{R}^{+}=$ $(0, \infty)$ and $x>0$. A classical inequality, due to Hardy et al. [1], states that

$$
\|H(f)\|_{L^{p}\left(\mathbb{R}^{+}\right)} \leq \frac{p}{p-1}\|f\|_{L^{p}\left(\mathbb{R}^{+}\right)}
$$

holds for $1<p<\infty$ and the constant $p /(p-1)$ is the best possible.

The Hardy integral inequality has received considerable attention. A number of papers involved its alternative proofs, generalizations, variants, and applications. Among numerous papers dealing with such inequalities, we choose to refer to the papers [2-4].

Let $f$ be a locally integrable function on $\mathbb{R}^{n}, 0 \leq \beta<$ $n$. In [5], Fu et al. defined $n$-dimensional fractional Hardy operators $\mathscr{H}_{\beta}$ :

$$
\mathscr{H}_{\beta} f(x)=\frac{1}{|x|^{n-\beta}} \int_{|y|<|x|} f(y) d y .
$$

When $\beta=0, \mathscr{H}_{\beta}$ is just $n$-dimensional Hardy operators $\mathscr{H}$; see [3] for more details.
It is well known that averaging operators play an important role in harmonic analysis. For example, the HardyLittlewood maximal operators control many kinds of operators in analysis. Therefore, the study of Hardy operators is meaningful and has been fully discussed (see [4, 6-11]). In 2012, Zhao et al. [11] have shown the following results: when $b \in B M O\left(\mathbb{R}^{n}\right)$, (1) $\mathscr{H}$ maps $H^{1}\left(\mathbb{R}^{n}\right)$ into $L^{1}\left(\mathbb{R}^{n}\right) ;(2)[b, \mathscr{H}]$ maps $H^{1}\left(\mathbb{R}^{n}\right)$ into $L^{1}\left(\mathbb{R}^{n}\right)$ was false; (3) $[b, \mathscr{H}]$ maps $H^{1}\left(\mathbb{R}^{n}\right) L^{1, \infty}\left(\mathbb{R}^{n}\right)$. Furthermore, (3) is also true for $b \in$ $C \dot{M} O^{q}\left(\mathbb{R}^{n}\right), 1 \leq q<\infty$. Recently, fractional Hardy operators were studied by many authors (see $[5,12,13])$. In 2007, Fu et al. [5] have obtained that the commutator $\left[b, \mathscr{H}_{\beta}\right]$ is bounded from $\dot{K}_{q_{1}}^{\gamma, p_{1}}\left(\mathbb{R}^{n}\right)$ to $\dot{K}_{q_{2}}^{\gamma, p_{2}}\left(\mathbb{R}^{n}\right)$, where $\gamma<n\left(1-1 / q_{1}\right)$. In 2013, Lu et al. [13] have proved that fractional Hardy operators $H_{\beta}$ map $L^{p}\left(\mathbb{R}^{n}\right)$ into $L^{q}\left(\mathbb{R}^{n}\right)$, where $1<p \leq n / \beta, 1 / q=(1 / p)-$ $(\beta / n)$, and $\left\|\mathscr{H}_{\beta}\right\|_{L^{1} \rightarrow L^{(n /(n-\beta)), \infty}}=1$. Inspired by the above, we consider the endpoint estimates for fractional Hardy operators and their commutators on Hardy-type spaces.

This paper is organized as follows. In the second section, we give the $\left(H^{p}\left(\mathbb{R}^{n}\right), L^{q}\left(\mathbb{R}^{n}\right)\right)$ bounds of fractional Hardy operators. In the third section, we obtain the estimates for commutators of fractional Hardy operators on Hardy spaces. In Section 4, we consider the case on Herz-type Hardy spaces. In Section 5, we obtain the estimate for multilinear commutators of fractional Hardy operators. 
Throughout this paper, $C_{1} \sim C_{2}$ denotes that $C_{1}$ is equivalent to $C_{2}$, which means there exist two positive constants $c_{1}$ and $c_{2}$ such that $c_{1} C_{1} \leq C_{2} \leq c_{2} C_{1}$. For $1<p$, and $p^{\prime}<\infty$, $p$ and $p^{\prime}$ are conjugate indices; that is, $(1 / p)+\left(1 / p^{\prime}\right)=1$.

Let us introduce some definitions below.

Definition 1. Let $1 \leq q<\infty$ and $b \in L_{\text {loc }}\left(\mathbb{R}^{n}\right)$. One says that $b \in C \dot{M} O^{q}\left(\mathbb{R}^{n}\right)$ if and only if

$$
\sup _{r>0}\left(\frac{1}{|B(0, r)|} \int_{B(0, r)}\left|b(x)-b_{B(0, r)}\right|^{q} d x\right)^{1 / q}<\infty,
$$

where $b_{B(0, r)}=(1 /|B(0, r)|) \int_{B(0, r)} b(x) d x$. The $C \dot{M} O^{q}$ norm of $b$ is defined by

$\|b\|_{C \dot{M} O^{q}\left(\mathbb{R}^{n}\right)}$

$$
=\sup _{r>0}\left(\frac{1}{|B(0, r)|} \int_{B(0, r)}\left|b(x)-b_{B(0, r)}\right|^{q} d x\right)^{1 / q}<\infty .
$$

Remark 2. When $1 \leq q<\infty, B M O\left(\mathbb{R}^{n}\right) \subseteq C \dot{M} O^{q}\left(\mathbb{R}^{n}\right)$; when $1 \leq p<q<\infty, C \dot{M} O^{q}\left(\mathbb{R}^{n}\right) \subseteq C \dot{M} O^{p}\left(\mathbb{R}^{n}\right)$. We choose to refer to papers $[5,9]$.

Definition 3. The Lipschitz space $\operatorname{Lip}_{\alpha}\left(\mathbb{R}^{n}\right)$ is the space of functions $f$ satisfying

$$
\|f\|_{\operatorname{Lip}_{\alpha}\left(\mathbb{R}^{n}\right)}:=\sup _{x, h \in \mathbb{R}^{n}, h \neq 0} \frac{|f(x+h)-f(x)|}{|h|^{\alpha}}<\infty,
$$

where $0<\alpha \leq 1$.

Remark 4. When $0<\alpha<1, \operatorname{Lip}_{\alpha}\left(\mathbb{R}^{n}\right)=\dot{\Lambda}_{\alpha}\left(\mathbb{R}^{n}\right)$, where $\dot{\wedge}_{\alpha}\left(\mathbb{R}^{n}\right)$ is the homogeneous Besov-Lipschitz space.

Definition 5 (see [14]). Let $0<p \leq 1$; a function is called $a(p, \infty, s)$-atom, where $s \geq[n(1 / p-1)]$, if it satisfies the following conditions:

$$
\begin{aligned}
& \text { (1) } \operatorname{supp}(a) \subset B\left(x_{0}, r\right) \text {; } \\
& \text { (2) }\|a\|_{L^{\infty}} \leq\left|B\left(x_{0}, r\right)\right|^{-1 / p} \\
& \text { (3) } \int_{\mathbb{R}^{n}} a(x) x^{\gamma} d x=0 \text {, where } 0 \leq|\gamma| \leq s .
\end{aligned}
$$

As a proper subspace of $L^{p}\left(\mathbb{R}^{n}\right)$, the atomic Hardy space $H^{p}\left(\mathbb{R}^{n}\right)$ is defined by

$$
\begin{aligned}
H^{p} & \left(\mathbb{R}^{n}\right) \\
& =\left\{f \in S^{\prime}\left(\mathbb{R}^{n}\right): f(x) \stackrel{S^{\prime}}{=} \sum_{k} \lambda_{k} a_{k}(x), \sum_{k}\left|\lambda_{k}\right|^{p}<\infty\right\},
\end{aligned}
$$

where each $a_{k}$ is a $(p, \infty, s)$-atom and $f$ is a tempered distribution. Set $H^{p}\left(\mathbb{R}^{n}\right)$ norm of $f$ by

$$
\|f\|_{H^{p}\left(\mathbb{R}^{n}\right)}:=\inf \left\{\left(\sum_{k}^{\infty}\left|\lambda_{k}\right|^{p}\right)^{1 / p}\right\}
$$

where the infimum has taken over all the decompositions of $f=\sum_{k} \lambda_{k} a_{k}$ as above.

Given a positive integer $m$ and $1 \leq i \leq m$, we denote by $C_{i}^{m}$ the family of all finite subsets $\sigma=\{\sigma(1), \sigma(2), \ldots, \sigma(i)\}$ of $\{1,2, \ldots, m\}$ of $j$ different elements. For $\sigma \in C_{i}^{m}$, set $\sigma^{c}=$ $\{1, \ldots, m\} \backslash \sigma$. For $\vec{b}=\left(b_{1}, b_{2}, \ldots, b_{m}\right)$ and $\sigma=\left\{\sigma_{1}, \sigma_{2}, \ldots, \sigma_{i}\right\} \in$ $C_{j}^{m}$, set $\vec{b}_{\sigma}=\left(b_{\sigma_{1}}, \ldots, b_{\sigma_{j}}\right), b_{\sigma}=b_{\sigma_{1}} \cdots b_{\sigma_{j}}$, and $\left\|\vec{b}_{\sigma}\right\|_{\operatorname{Lip}_{\alpha}\left(\mathbb{R}^{n}\right)}=$ $\left\|b_{\sigma_{1}}\right\|_{\operatorname{Lip}_{\alpha}\left(\mathbb{R}^{n}\right)} \cdots\left\|b_{\sigma_{i}}\right\|_{\operatorname{Lip}_{\alpha}\left(\mathbb{R}^{n}\right)}$.

Definition 6. Let $b_{i}(i=1,2, \ldots, m)$ be a locally integrable functions, and $0<p \leq 1$. A bounded measurable function $a$ on $\mathbb{R}^{n}$ is called $a(p, \vec{b})$-atom, if

(i) supp $a \subset B=B\left(x_{0}, r\right)$,

(ii) $\|a\|_{L^{\infty}} \leq\left|B\left(x_{0}, r\right)\right|^{-1 / p}$,

(iii) $\int_{B} a(y) d y=\int_{B} a(y) \prod_{j \in \sigma} b_{j} d y=0$ for any $\sigma \in C_{i}^{m}, 1 \leq$ $i \leq m$.

A temperate distribution (see $[15,16]) f$ is said to belong to $H_{\vec{b}}^{p}\left(\mathbb{R}^{n}\right)$, if, in the Schwartz distribution sense, it can be written as

$$
f(x)=\sum_{i} \lambda_{i} a_{i}(x)
$$

where $a_{i}$ is $(p, \vec{b})$-atom, $\lambda_{i} \in C$, and $\sum_{i}\left|\lambda_{i}\right|^{p}<\infty$. Moreover, $\|f\|_{H_{b}^{p}}=\inf \left(\sum_{i}\left|\lambda_{i}\right|^{p}\right)^{1 / p}$, where the infimum has taken over all the decompositions of $f$ as above.

Remark 7. When $m=1, H_{\vec{b}}^{1}\left(\mathbb{R}^{n}\right)=H_{b}^{1}\left(\mathbb{R}^{n}\right)$.

Let $B_{k}=\left\{x \in \mathbb{R}:|x|<2^{k}\right\}$ and $E_{k}=B_{k} \backslash B_{k-1}$ for $k \in \mathbb{Z}$. Denote $\chi_{k}=\chi_{E_{k}}$.

Definition 8. Let $\gamma \in \mathbb{R}, 0<p$ and $q \leq \infty$.

(i) The homogeneous Herz space $\dot{K}_{q}^{\gamma, p}\left(\mathbb{R}^{n}\right)$ is defined by

$$
\dot{K}_{q}^{\gamma, p}\left(\mathbb{R}^{n}\right)=\left\{f: f \in L_{\text {loc }}^{q}\left(\mathbb{R}^{n} \backslash\{0\}\right),\|f\|_{\dot{K}_{q}^{\gamma, p}}<\infty\right\},
$$

where

$$
\|f\|_{\dot{K}_{q}^{\gamma, p}\left(\mathbb{R}^{n}\right)}=\left\{\sum_{k \in \mathbb{Z}} 2^{k \gamma p}\left\|f \chi_{k}\right\|_{L^{q}\left(\mathbb{R}^{n}\right)}^{p}\right\}^{1 / p} .
$$

(ii) The nonhomogeneous Herz space $K_{q}^{\gamma, p}\left(\mathbb{R}^{n}\right)$ is defined by

$$
K_{q}^{\gamma, p}\left(\mathbb{R}^{n}\right)=\left\{f: f \in L_{\text {loc }}^{q}\left(\mathbb{R}^{n} \backslash\{0\}\right),\|f\|_{\dot{K}_{q}^{\gamma, p}}<\infty\right\},
$$

where

$$
\|f\|_{K_{q}^{\gamma, p}\left(\mathbb{R}^{n}\right)}=\left\{\sum_{k=0}^{\infty} 2^{k \gamma p}\left\|f \chi_{k}\right\|_{L^{q}\left(\mathbb{R}^{n}\right)}^{p}\right\}^{1 / p}
$$


Remark 9. When $0<q \leq \infty, \dot{K}_{q}^{0, q}\left(\mathbb{R}^{n}\right)=K_{q}^{0, q}\left(\mathbb{R}^{n}\right)=$ $L^{q}\left(\mathbb{R}^{n}\right)$, and $\dot{K}_{q}^{\gamma / q}\left(\mathbb{R}^{n}\right)=L_{|x|^{\gamma}}^{q}\left(\mathbb{R}^{n}\right)$.

Definition 10 (see [15]). Let $\gamma \in \mathbb{R}$, and $0<p<q<\infty$.

(i) The homogeneous Herz-type Hardy space $H \dot{K}_{q}^{\gamma, p}\left(\mathbb{R}^{n}\right)$ is defined by

$$
H \dot{K}_{q}^{\gamma, p}\left(\mathbb{R}^{n}\right)=\left\{f \in S^{\prime}\left(\mathbb{R}^{n}\right): G(f) \in \dot{K}_{q}^{\gamma, p}\left(\mathbb{R}^{n}\right)\right\},
$$

and we define $\|f\|_{H \dot{K}_{q}^{\gamma, p}\left(\mathbb{R}^{n}\right)}=\|G(f)\|_{\dot{K}_{q}^{\gamma, p}\left(\mathbb{R}^{n}\right)}$.

(ii) The nonhomogeneous Herz-type Hardy space $H K_{q}^{\gamma, p}\left(\mathbb{R}^{n}\right)$ is defined by

$$
H K_{q}^{\gamma, p}\left(\mathbb{R}^{n}\right)=\left\{f \in S^{\prime}\left(\mathbb{R}^{n}\right): G(f) \in K_{q}^{\gamma, p}\left(\mathbb{R}^{n}\right)\right\},
$$

and we define $\|f\|_{H K_{q}^{\gamma, p}\left(\mathbb{R}^{n}\right)}=\|G(f)\|_{K_{q}^{\gamma, p}\left(\mathbb{R}^{n}\right)}$.

Remark 11. When $0<p<\infty, H \dot{K}_{p}^{0, p}\left(\mathbb{R}^{n}\right)=K_{p}^{0, p}\left(\mathbb{R}^{n}\right)=$ $H^{p}\left(\mathbb{R}^{n}\right)$ and $H \dot{K}_{p}^{\gamma / p}\left(\mathbb{R}^{n}\right)=L_{|x|^{p}}^{p}\left(\mathbb{R}^{n}\right)$. And when $1<q<$ $\infty$, we know that $H \dot{K}_{q}^{\gamma, p}\left(\mathbb{R}^{n}\right)=\dot{K}_{q}^{\gamma, p}\left(\mathbb{R}^{n}\right)$ and $H K_{q}^{\gamma, p}\left(\mathbb{R}^{n}\right)=$ $K_{q}^{\gamma, p}\left(\mathbb{R}^{n}\right)$, where $-n / q<\gamma<n(1-1 / q)$. However, when $\gamma \geq$ $n(1-1 / q), H \dot{K}_{q}^{\gamma, p}\left(\mathbb{R}^{n}\right) \neq \dot{K}_{q}^{\gamma, p}\left(\mathbb{R}^{n}\right)$ and $H K_{q}^{\gamma, p}\left(\mathbb{R}^{n}\right) \neq K_{q}^{\gamma, p}\left(\mathbb{R}^{n}\right)$ (see $[17,18])$.

\section{2. $\left(H^{p}\left(\mathbb{R}^{n}\right), L^{q}\left(\mathbb{R}^{n}\right)\right)$ Bounds of Fractional Hardy Operators}

Theorem 12. Let $0<p \leq 1$ and $1 / q=1 / p-\beta / n$. $\mathscr{H}_{\beta}$ maps $H^{p}\left(\mathbb{R}^{n}\right)$ into $L^{q}\left(\mathbb{R}^{n}\right)$.

Proof. Assume that $a$ is an atom of $H^{p}\left(\mathbb{R}^{n}\right)$ and satisfies the following conditions: (i) $\operatorname{supp}(a) \subset B\left(x_{0}, r\right)$, (ii) $\|a\|_{L^{\infty}} \leq$ $\left|B\left(x_{0}, r\right)\right|^{-1 / p}$, and (iii) $\int a(x) x^{\gamma} d x=0$, where $0 \leq|\gamma| \leq s$, $s \geq[n(1 / p-1)]$. We now take $\widetilde{a}(x)=a\left(x+x_{0}\right)$; then $\widetilde{a}$ satisfies: (i) $\operatorname{supp}(\widetilde{a}) \subset B(0, r)$, (ii) $\|\widetilde{a}\|_{L^{\infty}} \leq|B(0, r)|^{-1 / p}$, and (iii) $\int \widetilde{a}(x) d x=0$.

Suppose that supp $\tilde{a} \subset B(0, r)$ for $r>0$. Consider

$$
\begin{aligned}
\int_{\mathbb{R}^{n}} & \left|\mathscr{H}_{\beta}(\widetilde{a})(x)\right|^{q} d x \\
= & \int_{\mathbb{R}^{n}}\left|\frac{1}{|x|^{n-\beta}} \int_{|y|<|x|} \tilde{a}(y) d y\right|^{q} d x \\
= & \int_{B(0, r)}\left|\frac{1}{|x|^{n-\beta}} \int_{|y|<|x|} \tilde{a}(y) d y\right|^{q} d x \\
& +\int_{\mathbb{R}^{n} \backslash B(0, r)}\left|\frac{1}{|x|^{n-\beta}} \int_{|y|<|x|} \tilde{a}(y) d y\right|^{q} d x \\
= & \int_{B(0, r)}\left|\frac{1}{|x|^{n-\beta}} \int_{\{|y|<|x|\} \cap B(0, r)} \tilde{a}(y) d y\right|^{q} d x \\
& +\int_{\mathbb{R}^{n} \backslash B(0, r)}\left|\frac{1}{|x|^{n-\beta}} \int_{\{|y|<|x|\} \cap B(0, r)} \tilde{a}(y) d y\right|^{q} d x \\
:= & I_{1}+I_{2},
\end{aligned}
$$

where we used the condition $\operatorname{supp}(a) \subset B(0, r)$. For $I_{1}$, we have the following estimate

$$
\begin{aligned}
I_{1} & \leq \int_{B(0, r)} \frac{1}{|x|^{(n-\beta) q}}\left(\int_{\{|y|<|x|\} \cap B(0, r)}|\widetilde{a}(y)| d y\right)^{q} d x \\
& \leq \int_{B(0, r)} \frac{1}{|x|^{(n-\beta) q}} \frac{1}{|B(0, r)|^{q / p}}\left(\int_{|y|<|x|} d y\right)^{q} d x \\
& \leq C \frac{1}{|B(0, r)|^{q / p}} \int_{B(0, r)} \frac{1}{|x|^{(n-\beta) q}|x|^{n q} d x} \\
& \leq C \frac{1}{|B(0, r)|^{q / p}} \int_{B(0, r)}|x|^{\beta \beta} d x \\
& \leq C \frac{1}{r^{n q / p}} \cdot r^{n+\beta q} \\
& \leq C<\infty,
\end{aligned}
$$

where we used the condition $\|\widetilde{a}\|_{L^{\infty}} \leq|B(0, r)|^{-1 / p}$ and $1 / q=$ $(1 / p)-(\beta / n)$. For $I_{2}$, since $x \in \mathbb{R}^{n} \backslash B(0, r)$, we have $\left\{y \in \mathbb{R}^{n}\right.$ : $|y|<|x|\} \supset\left\{y \in \mathbb{R}^{n}: y \in B(0, r)\right\}$. Then

$$
\begin{aligned}
I_{2} & =\int_{\mathbb{R}^{n} \backslash B(0, r)}\left|\frac{1}{|x|^{n-\beta}} \int_{\{|y|<|x|\} \cap B(0, r)} \tilde{a}(y) d y\right|^{q} d x \\
& =\int_{\mathbb{R}^{n} \backslash B(0, r)}\left|\frac{1}{|x|^{n-\beta}} \int_{B(0, r)} \tilde{a}(y) d y\right|^{q} d x=0 .
\end{aligned}
$$

The proof is completed.

\section{Estimates for Commutators of Fractional Hardy Operators on Hardy Spaces}

Definition 13 (see $[5,19])$. Let $b$ be a locally integrable function on $\mathbb{R}^{n}$. The commutator of $n$-dimensional Hardy operators is defined by

$$
[b, \mathscr{H}] f=b \mathscr{H} f-\mathscr{H}(b f) .
$$

Meanwhile, the commutators of $n$-dimensional fractional Hardy operators are defined by

$$
\left[b, \mathscr{H}_{\beta}\right] f=b \mathscr{H}_{\beta} f-\mathscr{H}_{\beta}(b f) .
$$

In general, the properties of commutator are worse than those of the operators themselves (e.g., the Hardy operators [11] and the singular integral operators [20]). Therefore, when $b$ is in $\operatorname{Lip}_{\alpha}\left(\mathbb{R}^{n}\right)$, we prove that $\left[b, \mathscr{H}_{\beta}\right]$ is not bounded from $H^{1}\left(\mathbb{R}^{n}\right)$ to $L^{n /(n-\beta-\alpha)}\left(\mathbb{R}^{n}\right)$. Furthermore, we conclude that the commutator maps from $H^{1}\left(\mathbb{R}^{n}\right)$ to $L^{n /(n-\beta-\alpha), \infty}\left(\mathbb{R}^{n}\right)$ and the commutator maps $H_{b}^{p}\left(\mathbb{R}^{n}\right)$ into $L^{q}\left(\mathbb{R}^{n}\right)$, where $0<p \leq 1$ and $1 / q=1 / p-(\beta+\alpha) / n$.

Proposition 14. If $b \in \operatorname{Lip}_{\alpha}\left(\mathbb{R}^{n}\right), q=n /(n-\beta-\alpha)$ and $0<$ $q<\infty$, then $\left[b, \mathscr{H}_{\beta}\right]$ is not bounded from $H^{1}\left(\mathbb{R}^{n}\right)$ to $L^{q}\left(\mathbb{R}^{n}\right)$. 
Proof. We give the proof only for the case $n=1$, then $q=$ $1 /(1-\beta-\alpha)$. Taking $b(x)=\left(|x|^{\alpha}-2^{\alpha}\right) \chi_{(2, \infty)}(x)$ and $f_{0}(x)=$ $\chi_{(0,2)}(x)-\chi_{(-2,0)}(x)$, it is easy to see that $b \in \operatorname{Lip}_{\alpha}\left(\mathbb{R}^{n}\right)$. Then for $x>3$, we have the following estimate:

$$
\begin{aligned}
& \left|\left[b, \mathscr{H}_{\beta}\right] f(x)\right| \\
& \quad=\left|\frac{1}{|x|^{1-\beta}} \int_{0}^{x}(b(x)-b(y)) f_{0}(y) d y\right| \\
& \quad=\frac{1}{x^{1-\beta}} \int_{0}^{2}\left(x^{\alpha}-2^{\alpha}-0\right) \times 1 d y \geq \frac{C_{\alpha}}{x^{1-\beta-\alpha}} .
\end{aligned}
$$

Here $C_{\alpha}$ is the constant dependent on $\alpha$. So we get

$$
\int_{\mathbb{R}^{1}}\left|\left[b, \mathscr{H}_{\beta}\right] f(x)\right|^{q} d x \geq \int_{3}^{\infty}\left(\frac{2}{x^{1-\beta-\alpha}}\right)^{q} d x=\infty .
$$

Theorem 15. Let $b \in \operatorname{Lip}_{\alpha}\left(\mathbb{R}^{n}\right), q=n /(n-\beta-\alpha)$, and $0<$ $q<\infty$ then $\left[b, \mathscr{H}_{\beta}\right]$ maps $H^{1}\left(\mathbb{R}^{n}\right)$ into $L^{q, \infty}\left(\mathbb{R}^{n}\right)$.

Proof. It is enough to prove that

$$
\begin{aligned}
\lambda \mid & \left.\left\{x \in \mathbb{R}^{n}:\left|\left[b, \mathscr{H}_{\beta}\right](\widetilde{a})(x)\right|>\lambda\right\}\right|^{1 / q} \\
& \leq C\|b\|_{\operatorname{Lip}_{\alpha}\left(\mathbb{R}^{n}\right)}\|\widetilde{a}\|_{H^{1}\left(\mathbb{R}^{n}\right)}
\end{aligned}
$$

holds for any $\widetilde{a}$ defined in the proof of Theorem 12 and the constant $\mathrm{C}$ is independent of $\widetilde{a}$. Suppose that supp $\widetilde{a} \subset B(0, r)$ for $r>0$. Then

$$
\begin{aligned}
\left|\left\{x \in \mathbb{R}^{n}:\left|\left[b, \mathscr{H}_{\beta}\right](\widetilde{a})(x)\right|>\lambda\right\}\right|^{1 / q} \\
\leq \max \left\{1, n^{(1 / q)-1}\right\} \\
\quad \times\left\{\left|\left\{x \in B(0, r):\left|\left[b, \mathscr{H}_{\beta}\right](\widetilde{a})(x)\right|>\frac{\lambda}{2}\right\}\right|^{1 / q}\right. \\
\left.\quad+\left|\left\{x \in \mathbb{R}^{n} \backslash B(0, r):\left|\left[b, \mathscr{H}_{\beta}\right](\widetilde{a})(x)\right|>\frac{\lambda}{2}\right\}\right|^{1 / q}\right\} .
\end{aligned}
$$

For the first term, $|y|<|x|$ implies $|x-y| \leq|x|+|y| \leq 2|x|$; then we have

$$
\begin{aligned}
\lambda^{q} \mid & \left\{x \in B(0, r):\left|\left[b, \mathscr{H}_{\beta}\right](\widetilde{a})(x)\right|>\frac{\lambda}{2}\right\} \mid \\
\leq & C \int_{B(0, r)}\left|\left[b, \mathscr{H}_{\beta}\right](\widetilde{a})(x)\right|^{q} d x \\
\leq & C \int_{B(0, r)}\left|\frac{1}{|x|^{n-\beta}} \int_{|y|<|x|} \tilde{a}(y)(b(x)-b(y)) d y\right|^{q} d x \\
\leq & C\|b\|_{\operatorname{Lip}_{\alpha}\left(\mathbb{R}^{n}\right)}^{q} \\
& \times \int_{B(0, r)}\left(\frac{1}{|x|^{n-\beta}} \int_{|y|<|x|}|\widetilde{a}(y)||x-y|^{\alpha} d y\right)^{q} d x \\
\leq & C\|b\|_{\operatorname{Lip}_{\alpha}\left(\mathbb{R}^{n}\right)}^{q} \int_{B(0, r)} \frac{1}{|B(0, r)|^{q}} \\
& \times\left(\frac{1}{|x|^{n-\beta}} \int_{|y|<|x|}|x-y|^{\alpha} d y\right)^{q} d x
\end{aligned}
$$

$$
\begin{aligned}
& \leq C\|b\|_{\operatorname{Lip}_{\alpha}\left(\mathbb{R}^{n}\right)}^{q} \frac{1}{|B(0, r)|^{q}} \int_{B(0, r)}|x|^{\beta q+\alpha q} d x \\
& \leq C\|b\|_{\operatorname{Lip}_{\alpha}\left(\mathbb{R}^{n}\right)}^{q} r^{-n q} \cdot r^{n+\alpha q+\beta q} \\
& =C\|b\|_{\operatorname{Lip}_{\alpha}\left(\mathbb{R}^{n}\right)}^{q}
\end{aligned}
$$

where $q=n /(n-\beta-\alpha)$. For the last term, by $x \in \mathbb{R}^{n} \backslash B(0, r)$, we have

$$
\begin{aligned}
& \left|\frac{1}{|x|^{n-\beta}} \int_{|y|<r}(b(x)-b(y)) \tilde{a}(y) d y\right| \\
& \quad \leq \frac{1}{|x|^{n-\beta}} \int_{|y|<r}|b(x)-b(y)||\tilde{a}(y)| d y \\
& \quad \leq C\|b\|_{\operatorname{Lip}_{\beta}\left(\mathbb{R}^{n}\right)} \frac{1}{|x|^{n-\beta}} \int_{|y|<r}|x-y|^{\alpha} \frac{1}{|B(0, r)|} d y \\
& \quad \leq C\|b\|_{\operatorname{Lip}_{\beta}\left(\mathbb{R}^{n}\right)} \frac{1}{|x|^{n-\beta}} \int_{|y|<r}|x|^{\alpha} \frac{1}{|B(0, r)|} d y \\
& \quad \leq C\|b\|_{\operatorname{Lip}_{\beta}\left(\mathbb{R}^{n}\right)} \frac{1}{|x|^{n-\beta-\alpha}} .
\end{aligned}
$$

So we obtain that

$$
\begin{aligned}
& \left|\left\{x \in \mathbb{R}^{n} \backslash B(0, r):\left|\left[b, \mathscr{H}_{\beta}\right](\widetilde{a})(x)>\frac{\lambda}{2}\right|\right\}\right| \\
& \quad \leq\left|\left\{x \in \mathbb{R}^{n} \backslash B(0, r): C\|b\|_{\operatorname{Lip}_{\beta}\left(\mathbb{R}^{n}\right)} \frac{1}{|x|^{n-\beta-\alpha}}>\lambda\right\}\right| \\
& \quad=C \int_{r}^{\left(C\|b\|_{\operatorname{Lip}_{\beta}\left(\mathbb{R}^{n}\right)} / \lambda\right)^{1 /(n-\alpha-\beta)}} t^{n-1} d t \\
& \quad \leq C\left(\frac{\|b\|_{\operatorname{Lip}_{\beta}\left(\mathbb{R}^{n}\right)}}{\lambda}\right)^{n /(n-\alpha-\beta)} \\
& \quad=C\left(\frac{\|b\|_{\operatorname{Lip}_{\beta}\left(\mathbb{R}^{n}\right)}}{\lambda}\right)^{q} .
\end{aligned}
$$

Combining all the above estimates, we complete the proof of Theorem 15.

Theorem 16. Let $b \in \operatorname{Lip}_{\alpha}\left(\mathbb{R}^{n}\right), 0<p \leq 1$ and $1 / q=1 / p-$ $(\beta+\alpha) / n$ and $0<q<\infty$; then $\left[b, \mathscr{H}_{\beta}\right]$ maps $H_{b}^{p}\left(\mathbb{R}^{n}\right)$ into $L^{q}\left(\mathbb{R}^{n}\right)$.

Proof. Similar to the proof above, suppose that supp $\widetilde{a} \subset$ $B(0, r)$ for $r>0$. Consider

$$
\begin{aligned}
\int_{\mathbb{R}^{n}} & \left|\left[b, \mathscr{H}_{\beta}\right](\widetilde{a})(x)\right|^{q} d x \\
= & \int_{\mathbb{R}^{n}}\left|\frac{1}{|x|^{n-\beta}} \int_{|y|<|x|} \tilde{a}(y)(b(x)-b(y)) d y\right|^{q} d x \\
= & \int_{B(0, r)} \mid \frac{1}{|x|^{n-\beta}} \\
& \quad \times\left.\int_{\{|y|<|x|\} \cap B(0, r)} \tilde{a}(y)(b(x)-b(y)) d y\right|^{q} d x
\end{aligned}
$$




$$
\begin{aligned}
& +\int_{\mathbb{R}^{n} \backslash B(0, r)} \mid \frac{1}{|x|^{n-\beta}} \int_{\{|y|<|x|\} \cap B(0, r)} \tilde{a}(y) \\
& \quad \times\left.(b(x)-b(y)) d y\right|^{q} d x \\
& \leq \int_{B(0, r)} \mid \frac{1}{|x|^{n-\beta}} \\
& \quad \times\left.\int_{\{|y|<|x|\} \cap B(0, r)} \tilde{a}(y)(b(x)-b(y)) d y\right|^{q} d x \\
& +C \int_{\mathbb{R}^{n} \backslash B(0, r)}\left|\frac{1}{|x|^{n-\beta}} \int_{\{|y|<|x|\} \cap B(0, r)} \tilde{a}(y) b(x) d y\right|^{q} d x \\
& \quad+C \int_{\mathbb{R}^{n} \backslash B(0, r)}\left|\frac{1}{|x|^{n-\beta}} \int_{\{|y|<|x|\} \cap B(0, r)} \tilde{a}(y) b(y) d y\right|^{q} d x \\
& :=J_{1}+J_{2}+J_{3} .
\end{aligned}
$$

For $J_{1}$, by the fact that $|y|<|x|$ implies $|x-y| \leq|x|+|y| \leq$ $2|x|$, we have that

$$
\begin{aligned}
J_{1}= & \int_{B(0, r)}\left|\frac{1}{|x|^{n-\beta}} \int_{\{|y|<|x|\} \cap B(0, r)} \tilde{a}(y)(b(x)-b(y)) d y\right|^{q} d x \\
\leq & C\|b\|_{\operatorname{Lip}_{\alpha}\left(\mathbb{R}^{n}\right)}^{q} \\
& \times\left.\left.\int_{B(0, r)}\left|\frac{1}{|x|^{n-\beta}} \frac{1}{|B(0, r)|^{1 / p}} \int_{|y|<|x|}\right| x\right|^{\alpha} d y\right|^{q} d x \\
\leq & \left.\left.C\|b\|_{\operatorname{Lip}_{\alpha}\left(\mathbb{R}^{n}\right)}^{q} \int_{B(0, r)}|| x\right|^{\beta+\alpha} \frac{1}{|B(0, r)|^{1 / p}}\right|^{q} d x \\
\leq & C\|b\|_{\operatorname{Lip}_{\alpha}\left(\mathbb{R}^{n}\right)}^{q}
\end{aligned}
$$

By $\int \widetilde{a}(y) d y=\int \widetilde{a}(y) b(y) d y=0$, we obtain that $J_{2}=J_{3}=0$. Then $\left[b, \mathscr{H}_{\beta}\right]$ maps $H_{b}^{p}\left(\mathbb{R}^{n}\right)$ into $L^{q}\left(\mathbb{R}^{n}\right)$.

Theorem 17. Let $b \in \operatorname{Lip}_{\alpha}\left(\mathbb{R}^{n}\right), 1 / q=1 / p-(\beta+\alpha) / n$, and $0<p \leq n /(n+\alpha) ; 0<q<\infty$, then the following two conditions are equivalent:

(i) $\left[b, \mathscr{H}_{\beta}\right]$ is bounded from $H^{p}\left(\mathbb{R}^{n}\right)$ to $L^{q}\left(\mathbb{R}^{n}\right)$;

(ii) for all $a(p, \infty, 0)$-atom, $\int_{\mathbb{R}^{n}} a(y) b(y) d y=0$.

Proof. By Theorem 16, it is clear that (ii) $\Rightarrow$ (i) is obvious. We only need to prove (i) $\Rightarrow$ (ii). We assume that supp $a \subset B=$ $B\left(x_{0}, r\right)$ for $r>0$. Let

$$
\begin{gathered}
v_{1}(x)=\chi_{B}\left[b, \mathscr{H}_{\beta}\right] a(x), \\
v_{2}(x)=\chi_{\mathbb{R}^{n} \backslash B}(x) b(x) \frac{1}{|x|^{n-\beta}} \int_{B} a(y) d y, \\
v_{3}(x)=\chi_{\mathbb{R}^{n} \backslash B}(x) \frac{1}{|x|^{n-\beta}} \int_{B} a(y) b(y) d y .
\end{gathered}
$$

Then

$$
\left[b, \mathscr{H}_{\beta}\right] a(x)=v_{1}(x)+v_{2}(x)-v_{3}(x) .
$$

Obviously, $\left\|v_{1}\right\|_{q} \leq C$, and $\left\|v_{2}\right\|_{q}=0$. For $N>2$,

$$
\begin{aligned}
C & \geq\left\|v_{3}\right\|_{q} \\
& \geq\left|\int_{B} a(y) b(y) d y\right|\left\{\int_{r<\left|x-x_{0}\right| \leq N r} \frac{1}{|x|^{(n-\beta) q}} d x\right\}^{1 / q} .
\end{aligned}
$$

By $0<p \leq n /(n+\alpha)$, we have $(n-\beta) q \leq n$.

When $N \rightarrow \infty, \int_{r<\left|x-x_{0}\right| \leq N r}\left(1 /|x|^{(n-\beta) q}\right) d x \rightarrow \infty$; then

$$
\int_{B} a(y) b(y) d y=0 .
$$

When $b$ is in $C \dot{M} O\left(\mathbb{R}^{n}\right)$, the commutator $\left[b, \mathscr{H}_{\beta}\right]$ has the similar properties.

Proposition 18. If $b \in B M O\left(\mathbb{R}^{n}\right)$ and $q=n /(n-\beta)$, then $\left[b, \mathscr{H}_{\beta}\right]$ is not bounded from $H^{1}\left(\mathbb{R}^{n}\right)$ to $L^{q}\left(\mathbb{R}^{n}\right)$.

Theorem 19. Let $b \in C \dot{M} O^{\max \{s q, s\}}\left(\mathbb{R}^{n}\right)$ and $q=n /(n-\beta)$, $1<s<\infty$, then $\left[b, \mathscr{H}_{\beta}\right]$ maps $H^{1}\left(\mathbb{R}^{n}\right)$ into $L^{q, \infty}\left(\mathbb{R}^{n}\right)$.

Remark 20. In 2013, Yu and Lu [21] have given the analogous results of Proposition 18 and Theorem 19.

Theorem 21. Let $b \in C \dot{M} O^{\max \{s q, s\}}\left(\mathbb{R}^{n}\right), 0<p \leq 1$ and $1<$ $s<\infty, 1 / q=1 / p-\beta / n$; then $\left[b, \mathscr{H}_{\beta}\right]$ is bounded from $H_{b}^{p}\left(\mathbb{R}^{n}\right)$ to $L^{q}\left(\mathbb{R}^{n}\right)$.

Theorem 22. Let $b \in C \dot{M} O^{\max \{s q, s\}}\left(\mathbb{R}^{n}\right)$ and $1 / q=1 / p-\beta / n$, $1<s<\infty$, and $0<p \leq 1$; then the following two conditions are equivalent:

(i) $\left[b, \mathscr{H}_{\beta}\right]$ maps $H^{p}\left(\mathbb{R}^{n}\right)$ into $L^{q}\left(\mathbb{R}^{n}\right)$;

(ii) for all $a(p, \infty, 0)$-atom, $\int_{\mathbb{R}^{n}} a(y) b(y) d y=0$.

\section{Estimates for Commutators of Fractional Hardy Operators on Herz-Type Hardy Spaces}

The boundedness of $\left[b, \mathscr{H}_{\beta}\right]$ on the Herz spaces $\dot{K}_{q}^{\gamma, p}\left(\mathbb{R}^{n}\right)$ has been obtained as the following, where $\gamma<n\left(1-1 / q_{1}\right)$.

Proposition 23 (see [22]). Let $\beta \geq 0,0<p_{1} \leq p_{2}<\infty$, $1 / q_{2}=1 / q_{1}-(\beta+\alpha) / n, 1<q_{1}, q_{2}<\infty, 1 / q_{1}+1 / q_{1}^{\prime}=1$ and $b \in \operatorname{Lip}_{\alpha}\left(\mathbb{R}^{n}\right)$; then $\gamma<n / q_{1}^{\prime}$ implies that $\left[b, \mathscr{H}_{\beta}\right]$ is bounded from $\dot{K}_{q_{1}}^{\gamma, p_{1}}\left(\mathbb{R}^{n}\right)$ to $\dot{K}_{q_{2}}^{\gamma, p_{2}}\left(\mathbb{R}^{n}\right)$.

Proposition 24 (see [5]). Let $\beta \geq 0,0<p_{1} \leq p_{2}<\infty$, $1 / q_{2}=1 / q_{1}-\beta / n, 1<q_{1}<\infty, 1 / q_{1}+1 / q_{1}^{\prime}=1$ and $b \epsilon$ $C \dot{M} O^{\max \left(q_{2}, q_{1}^{\prime}\right)}\left(\mathbb{R}^{n}\right)$; then $\gamma<n / q_{1}^{\prime}$ implies that $\left[b, \mathscr{H}_{\beta}\right]$ is bounded from $\dot{K}_{q_{1}}^{\gamma, p_{1}}\left(\mathbb{R}^{n}\right)$ to $\dot{K}_{q_{2}}^{\gamma, p_{2}}\left(\mathbb{R}^{n}\right)$.

In this section, we discuss the case $\gamma \geq n\left(1-1 / q_{1}\right)$. 
Definition 25. Let $\gamma \in \mathbb{R}$ and $1<q<\infty$. A function is called central $(\gamma, q)$-atom, if it satisfies the following conditions:

(i) $\operatorname{supp} a \subset B(0, r)$;

(ii) $\|a\|_{q} \leq|B(0, r)|^{-\gamma / n}$;

(iii) $\int_{\mathbb{R}^{n}} a(x) x^{s} d x=0$, where $|s| \leq[\gamma-n(1-1 / q)]$.

Lemma 26 (see [18]). Let $0<p<\infty, 1<q<\infty$ and $\gamma \geq$ $n(1-1 / q)$. Consider that $f \in H \dot{K}_{q}^{\gamma, p}\left(\mathbb{R}^{n}\right)$ if and only if

$$
f=\sum_{k \in \mathbb{Z}} \lambda_{k} a_{k}, \quad \text { in the sense of } S^{\prime}\left(\mathbb{R}^{n}\right),
$$

where each $a_{k}$ is a central $(\gamma, q)$-atom with the support $B_{k}$ and $\sum_{k \in \mathbb{Z}}\left|\lambda_{k}\right|^{p}<\infty$. Moreover,

$$
\|f\|_{H \dot{K}_{q}^{\gamma, p}\left(\mathbb{R}^{n}\right)} \sim \inf \left\{\left(\sum_{k \in \mathbb{Z}}\left|\lambda_{k}\right|^{p}\right)^{1 / p}\right\}
$$

where the infimum has taken over all above decompositions of $f$.

Theorem 27. Let $b \in \operatorname{Lip}_{\alpha}\left(\mathbb{R}^{n}\right), \gamma \geq n\left(1-1 / q_{1}\right)+\alpha, 0<p<$ $\infty, 1<q_{1}, q_{2}<\infty, 1 / q_{2}=1 / q_{1}-(\beta+\alpha) / n$, then the following two conditions are equivalent:

(i) $\left[b, \mathscr{H}_{\beta}\right]$ maps $H \dot{K}_{q_{1}}^{\gamma, p}\left(\mathbb{R}^{n}\right)$ into $\dot{K}_{q_{2}}^{\gamma, p}\left(\mathbb{R}^{n}\right)$;

(ii) for all central $a\left(\gamma, q_{1}\right)$-atom, $\int_{\mathbb{R}^{n}} b(y) a(y) d y=0$.

Proof. (i) $\Rightarrow$ (ii). Let $a$ be a central $\left(\gamma, q_{1}\right)$-atom, with the support $B_{k}$. In fact, if $\operatorname{supp} a(x) \subset B(0, r)$, let $k \in \mathbb{Z}$ such that $2^{k}-1<r \leq 2^{k}$, and for $C\left(n, q_{1}, \alpha\right)=2^{-\gamma}$, set $a^{\prime}=C\left(n, q_{1}, \alpha\right) a$; then $a^{\prime}$ is a central $\left(\gamma, q_{1}\right)$-atom with the support $B_{k}$.

Similar to the proof of Theorem 16, we have

$$
\begin{gathered}
v_{1}(x)=\chi_{B_{k}}\left[b, \mathscr{H}_{\beta}\right] a(x), \\
v_{2}(x)=\chi_{\mathbb{R}^{n} \backslash B_{k}}(x) b(x) \frac{1}{|x|^{n-\beta}} \int_{B} a(y) d y, \\
v_{3}(x)=\chi_{\mathbb{R}^{n} \backslash B_{k}}(x) \frac{1}{|x|^{n-\beta}} \int_{B} a(y) b(y) d y .
\end{gathered}
$$

Then

$$
\left[b, \mathscr{H}_{\beta}\right] a(x)=v_{1}(x)+v_{2}(x)-v_{3}(x) .
$$

Using the fact that $\left[b, \mathscr{H}_{\beta}\right]$ maps $L^{\mathrm{q}_{1}}\left(\mathbb{R}^{n}\right)$ into $L^{q_{2}}\left(\mathbb{R}^{n}\right)$

$$
\begin{aligned}
\left\|v_{1}\right\|_{\dot{K}_{q}^{\gamma, p}\left(\mathbb{R}^{n}\right)} & \leq\left(\sum_{j=-\infty}^{k+1} 2^{j \gamma p}\left\|\chi_{j}\left(\left[b, \mathscr{H}_{\beta}\right] a\right)\right\|_{q_{2}}^{p}\right)^{1 / p} \\
& \leq C\left(\sum_{j=-\infty}^{k+1} 2^{j \gamma p}\|a\|_{q_{1}}^{p}\right)^{1 / p} \\
& \leq C .
\end{aligned}
$$

When $|x| \geq 2^{k+1}$, we have $\left\|v_{2}\right\|_{\dot{K}_{q_{2}}^{\gamma, p}\left(\mathbb{R}^{n}\right)}=0$. On the other hand, $\|a\|_{H \dot{K}_{q_{1}}^{\gamma, p}\left(\mathbb{R}^{n}\right)} \leq 1$, so $\left\|\left[b, \mathscr{H}_{\beta}\right] a\right\|_{\dot{K}_{q_{2}}^{\gamma, p}} \leq C$. Combining all the above estimates, we have $\left\|v_{3}\right\|_{\dot{K}_{q_{2}}^{\gamma, p}\left(\mathbb{R}^{n}\right)} \leq C$. For $N>3$, by $\gamma \geq$ $n\left(1-1 / q_{1}+\alpha\right)$, we have that

$$
\begin{aligned}
C^{p} & \geq \sum_{j=k+2}^{\infty} 2^{j \gamma p}\left(\int_{E_{j}}\left|\frac{1}{|x|^{n-\beta}} \int_{|y|<|x|} b(y) a(y) d y\right|^{q_{2}} d x\right)^{p / q_{2}} \\
& \geq \sum_{j=k+3}^{k+N} 2^{j \gamma p}\left(\left|\int_{\mathbb{R}^{n}} b(y) a(y) d y\right| \int_{E_{j}}|x|^{(\beta-n) q_{2}} d x\right)^{p / q_{2}} \\
& \geq C \sum_{j=k+3}^{k+N} 2^{j\left(\gamma-n\left(1-1 / q_{1}\right)+\alpha\right) p}\left|\int_{\mathbb{R}^{n}} b(y) a(y) d y\right|^{p} .
\end{aligned}
$$

Set $N \rightarrow \infty$; then

$$
\int_{\mathbb{R}^{n}} b(y) a(y) d y=0 .
$$

(ii) $\Rightarrow$ (i). Similar to the previous proof, by $\int_{\mathbb{R}^{n}} b(y) a(y) d y=0$, we obtain $\left\|v_{3}\right\|_{\dot{K}_{q_{2}}^{\gamma, p}\left(\mathbb{R}^{n}\right)}=0$. Combining $\left\|v_{1}\right\|_{\dot{K}_{q_{2}}^{\gamma, p}\left(\mathbb{R}^{n}\right)} \leq C$ and $\left\|v_{2}\right\|_{\dot{K}_{q_{2}}^{\gamma, p}\left(\mathbb{R}^{n}\right)}=0,\left[b, \mathscr{H}_{\beta}\right]$ maps $H \dot{K}_{q_{1}}^{\gamma, p}\left(\mathbb{R}^{n}\right)$ into $\dot{K}_{q_{2}}^{n\left(1-1 / q_{1}\right)+\beta+\alpha, p}\left(\mathbb{R}^{n}\right)$.

When $b$ is in $C \dot{M O}\left(\mathbb{R}^{n}\right)$, the commutator $\left[b, \mathscr{H}_{\beta}\right]$ also has the similar properties.

Theorem 28. Let $b \in C \dot{M} O^{\max \{s q, s\}}\left(\mathbb{R}^{n}\right), \gamma \geq n\left(1-1 / q_{1}\right), 1<$ $s<\infty, 0<p<\infty, 1<q_{1}, q_{2}<\infty$ and $1 / q_{2}=1 / q_{1}-\beta / n$; then the following two conditions are equivalent:

(i) $\left[b, \mathscr{H}_{\beta}\right]$ maps $H \dot{K}_{q_{1}}^{\gamma, p}\left(\mathbb{R}^{n}\right)$ into $\dot{K}_{q_{2}}^{\gamma, p}\left(\mathbb{R}^{n}\right)$;

(ii) for all central $a\left(\gamma, q_{1}\right)$-atom, $\int_{\mathbb{R}^{n}} b(y) a(y) d y=0$.

\section{Estimates for Multilinear Commutators of Fractional Hardy Operators}

Definition 29. The multilinear commutator of fractional Hardy operators is defined by

$$
\left[\vec{b}, \mathscr{H}_{\beta}\right]=\frac{1}{|x|^{n-\beta}} \int_{|y|<|x|} \prod_{i=1}^{m}\left(b_{i}(x)-b_{i}(y)\right) f(y) d y,
$$

where $\vec{b}=\left(b_{1}, b_{2}, \ldots, b_{m}\right)$ is a $m$-dimensional vector. When $m=0,\left[\vec{b}, \mathscr{H}_{\beta}\right]=\mathscr{H}_{\beta}$. When $m=1,\left[\vec{b}, \mathscr{H}_{\beta}\right]=\left[b, \mathscr{H}_{\beta}\right]$.

The study of multilinear operators is motivated by a mere quest to generalize the theory of linear operators and by their natural appearance in analysis (see [23-25]). In this section, we consider the multilinear commutators of fractional Hardy operators on Hardy spaces.

Theorem 30. Let $\vec{b}=\left(b_{1}, b_{2}, \ldots, b_{m}\right), b_{i} \in \operatorname{Lip}_{\alpha}\left(\mathbb{R}^{n}\right), 1 / q=$ $1 / p-(\beta+m \alpha) / n, 0<p \leq 1$ and $0<q<\infty$; then $\left[\vec{b}, \mathscr{H}_{\beta}\right]$ maps $H_{\vec{b}}^{p}\left(\mathbb{R}^{n}\right)$ to $L^{q}\left(\mathbb{R}^{n}\right)$. 
Journal of Function Spaces

7

Proof. Similar to the proof above, when $x \in \mathbb{R}^{n} \backslash B(0, r)$, we have

$$
\int_{\mathbb{R}^{n} \backslash B(0, r)}\left|\left[\vec{b}, \mathscr{H}_{\beta}\right](\widetilde{a})(x)\right|^{q} d x=0,
$$

so it is enough to prove that

$$
\left(\int_{B(0, r)}\left|\left[\vec{b}, \mathscr{H}_{\beta}\right](\widetilde{a})(x)\right|^{q} d x\right)^{1 / q} \leq C\|b\|_{\operatorname{Lip}_{\alpha}\left(\mathbb{R}^{n}\right)}\|\widetilde{a}\|_{H_{\vec{b}}^{1}}
$$

By $b_{i} \in \operatorname{Lip}_{\alpha}\left(\mathbb{R}^{n}\right)$, we obtain $\left|b_{i}(x)-b_{i}(y)\right| \leq\left\|b_{i}\right\|_{\operatorname{Lip}_{\alpha}\left(\mathbb{R}^{n}\right)} \mid x-$ $\left.y\right|^{\alpha}, i=1,2, \ldots, m$, and

$$
\begin{aligned}
& \left(\int_{B(0, r)}\left|\left[\vec{b}, \mathscr{H}_{\beta}\right](\widetilde{a})(x)\right|^{q} d x\right)^{1 / q} \\
& \leq\left(\int_{B(0, r)} \mid \frac{1}{|x|^{n-\beta}} \int_{|y|<|x|} \tilde{a}(y)\right. \\
& \left.\times\left.\prod_{i=1}^{m}\left(b_{i}(x)-b_{\mathrm{i}}(y)\right) d y\right|^{q} d x\right)^{1 / q} \\
& \leq \prod_{i=1}^{m}\left\|b_{i}\right\|_{\operatorname{Lip}_{\alpha}\left(\mathbb{R}^{n}\right)} \\
& \times\left(\int_{B(0, r)} \mid \frac{1}{|x|^{n-\beta}}\right. \\
& \left.\left.\times \int_{|y|<|x|}|\widetilde{a}(y)| \mid x-y\right)\left.\left.\right|^{m \alpha} d y\right|^{q} d x\right)^{1 / q} \\
& \leq C \prod_{i=1}^{m}\left\|b_{i}\right\|_{\operatorname{Lip}_{\alpha}\left(\mathbb{R}^{n}\right)} \\
& \times\left(\int_{B(0, r)} \mid \frac{1}{|x|^{n-\beta}}\right. \\
& \left.\times\left.\int_{|y|<|x|} r^{-n / p} \cdot 2|x|^{m \alpha} d y\right|^{q} d x\right)^{1 / q} \\
& \leq C \prod_{i=1}^{m}\left\|b_{i}\right\|_{\operatorname{Lip}_{\alpha}\left(\mathbb{R}^{n}\right)} \\
& \times\left(\int_{B(0, r)}\left|\frac{1}{|x|^{n-\beta}} \int_{|y|<|x|} r^{-n / p+m \alpha} d y\right|^{q} d x\right)^{1 / q} \\
& \leq C \prod_{i=1}^{m}\left\|b_{i}\right\|_{\operatorname{Lip}_{\alpha}\left(\mathbb{R}^{n}\right)}\left(\int_{B(0, r)}\left|r^{\beta} \cdot r^{-n / p+m \alpha}\right|^{q} d x\right)^{1 / q} \\
& \leq C \prod_{i=1}^{m}\left\|b_{i}\right\|_{\operatorname{Lip}_{\alpha}\left(\mathbb{R}^{n}\right)} \text {, }
\end{aligned}
$$

where $|x|<r$ and $1 / q=(1 / p)-((\beta+m \alpha) / n)$; we complete the proof of Theorem 30 .

Theorem 31. Let $\vec{b}=\left(b_{1}, b_{2}, \ldots, b_{m}\right), b_{i} \in \operatorname{Lip}_{\alpha}\left(\mathbb{R}^{n}\right), q=$ $n /(n-\beta-m \alpha)$, and $0<q<\infty$; then $\left[\vec{b}, \mathscr{H}_{\beta}\right]$ maps $H^{p}\left(\mathbb{R}^{n}\right)$ into $L^{q, \infty}\left(\mathbb{R}^{n}\right)$.

Proof. It is similar to the proof of Theorem 15.
When $b$ is in $C \dot{M} O\left(\mathbb{R}^{n}\right)$, we suppose the multilinear commutator of fractional Hardy operators maps $H^{1}\left(\mathbb{R}^{n}\right)$ into $L^{n /(n-\beta), \infty}$, but we cannot prove it. However, we get the following result.

Theorem 32. Let $\vec{b}=\left(b_{1}, b_{2}, \ldots, b_{m}\right)$ and $b_{i} \in$ $C \dot{M} O^{\max \left\{s_{i} q, s_{i}\right\}}\left(\mathbb{R}^{n}\right), 1 / q=1 / p-\beta / n, 0<p \leq 1,1<s_{i}<\infty$, then $\left[\vec{b}, \mathscr{H}_{\beta}\right]$ maps $H_{\vec{b}}^{p}\left(\mathbb{R}^{n}\right)$ into $L^{q}\left(\mathbb{R}^{n}\right)$.

Proof. Similar to the proof of Theorem 12, it is enough to prove that

$$
\left\|\left[\vec{b}, \mathscr{H}_{\beta}\right](\widetilde{a})(x)\right\|_{L^{q}} \leq C \prod_{i=1}^{m}\left\|b_{i}\right\|_{C \dot{M} O\left(\mathbb{R}^{n}\right)}\|\widetilde{a}\|_{H_{b}^{1}}
$$

where $\widetilde{a}$ is a center $(p, \vec{b})$-atom supported on a ball $B=$ $B(0, r)$. We write

$$
\begin{aligned}
{\left[\vec{b}, \mathscr{H}_{\beta}\right](\widetilde{a})(x) } & =\frac{1}{|x|^{n-\beta}} \int_{|y|<|x|} \tilde{a}(y) \prod_{i=1}^{m}\left(b_{i}(x)-b_{i}(y)\right) d y \\
= & \frac{1}{|x|^{n-\beta}} \int_{|y|<|x|} \tilde{a}(y) \sum_{i=0}^{m} \sum_{\sigma \in C_{i}^{m}} \prod_{i \in \sigma}\left(b_{i}(x)-\lambda_{i}\right) \\
= & \frac{1}{|x|^{n-\beta}} \prod_{i=1}^{m}\left(b_{i}(x)-\prod_{i}\right) \int_{|y|<|x|} \tilde{a}\left(\lambda_{i}-b_{i}(y)\right) d y \\
& +\sum_{i=1}^{m-1} \sum_{\sigma \in C_{i}^{m}} \frac{1}{|x|^{n-\beta}} \prod_{i \in \sigma}\left(b_{i}(x)-\lambda_{i}\right) \\
& +\frac{1}{|x|^{n-\beta}} \int_{|y|<|x|} \widetilde{a}(y) \prod_{i=1}^{m}\left(\lambda_{i}-b_{i}(y)\right) d y \\
:= & K_{1}+K_{2}+K_{3}, \\
& \times \int_{|y|<|x|} \tilde{a}(y) \prod_{i \in \sigma^{c}}\left(\lambda_{i}-b_{i}(y)\right) d y
\end{aligned}
$$

where $\lambda_{i}=\left(b_{i}\right)_{B}$. By Minkowski inequality, we have

$$
\begin{aligned}
\left\|\left[\vec{b}, \mathscr{H}_{\beta}\right](\widetilde{a})(x)\right\|_{L^{q}} \\
=\int_{\mathbb{R}^{n}}\left|\left[\vec{b}, \mathscr{H}_{\beta}\right](\widetilde{a})(x)\right|^{q} d x \\
\leq\left(\int_{\mathbb{R}^{n}}\left|K_{1}+K_{2}+K_{3}\right|^{q} d x\right)^{1 / q} \\
\leq\left(\int_{\mathbb{R}^{n}}\left|K_{1}\right|^{q} d x\right)^{1 / q}+\left(\int_{\mathbb{R}^{n}}\left|K_{2}\right|^{q} d x\right)^{1 / q} \\
\quad+\left(\int_{\mathbb{R}^{n}}\left|K_{3}\right|^{q} d x\right)^{1 / q}
\end{aligned}
$$




$$
\begin{aligned}
\leq & \left(\int_{B(0, r)}\left|K_{1}\right|^{q} d x\right)^{1 / q}+\left(\int_{\mathbb{R}^{n} \backslash B(0, r)}\left|K_{1}\right|^{q} d x\right)^{1 / q} \\
& +\left(\int_{B(0, r)}\left|K_{2}\right|^{q} d x\right)^{1 / q}+\left(\int_{\mathbb{R}^{n} \backslash B(0, r)}\left|K_{2}\right|^{q} d x\right)^{1 / q} \\
& +\left(\int_{B(0, r)}\left|K_{3}\right|^{q} d x\right)^{1 / q}+\left(\int_{\mathbb{R}^{n} \backslash B(0, r)}\left|K_{3}\right|^{q} d x\right)^{1 / q} \\
:= & K_{11}+K_{12}+K_{21}+K_{22}+K_{31}+K_{32} .
\end{aligned}
$$

For $x \in B(0, r)$, we have $|x|<r$; then

$$
\begin{aligned}
& K_{11}=\left(\int_{B(0, r)} \mid \frac{1}{|x|^{n-\beta}} \prod_{i=1}^{m}\left(b_{j}(x)-\lambda_{i}\right)\right. \\
& \left.\times\left.\int_{|y|<|x|} \tilde{a}(y) d y\right|^{q} d x\right)^{1 / q} \\
& \leq C\left(\int_{B(0, r)}\left|\frac{1}{|x|^{n-\beta}} \cdot \prod_{i=1}^{m}\right| b_{i}(x)-\left.\lambda_{i}|\cdot| x\right|^{n}\right. \\
& \left.\left.\cdot|B(0, r)|^{-1 / p}\right|^{q} d x\right)^{1 / q} \\
& \leq C r^{(\beta-n / p)}\left(\int_{B(0, r)} \prod_{i=1}^{m}\left|b_{i}(x)-\lambda_{i}\right|^{q} d x\right)^{1 / q} \\
& \leq C\left(\frac{1}{B(0, r)} \int_{B(0, r)} \prod_{i=1}^{m}\left|b_{i}(x)-\lambda_{i}\right|^{q} d x\right)^{1 / q} \\
& \leq C \prod_{i=1}^{m}\left\|b_{i}\right\|_{C \dot{M} O^{s_{i} q}\left(\mathbb{R}^{n}\right)}, \\
& K_{31}=\left(\int_{B(0, r)} \mid \frac{1}{|x|^{n-\beta}}\right. \\
& \left.\times\left.\int_{|y|<|x|} \tilde{a}(y) \prod_{i=1}^{m}\left(\lambda_{i}-b_{i}(y)\right) d y\right|^{q} d x\right)^{1 / q} \\
& \leq C\left(\left.\int_{B(0, r)}\left|\frac{1}{|x|^{n-\beta}} \cdot\right| B(0, r)\right|^{-1}\right. \\
& \left.\times\left.\int_{|y|<|x|} \prod_{i=1}^{m}\left(\lambda_{i}-b_{i}(y)\right) d y\right|^{q} d x\right)^{1 / q} \\
& \leq C\left(\left.\int_{B(0, r)}|| B(0, r)\right|^{-1 / p} \cdot|x|^{\beta} \cdot \frac{1}{B(0,|x|)}\right. \\
& \left.\times\left.\int_{|y|<|x|} \prod_{i=1}^{m}\left(\lambda_{i}-b_{i}(y)\right) d y\right|^{q} d x\right)^{1 / q}
\end{aligned}
$$

$$
\begin{aligned}
& \leq C\left(\left.\int_{B(0, r)}|| B(0, r)\right|^{-1 / p} \cdot|x|^{\beta}\right. \\
& \left.\left.\cdot \prod_{i=1}^{m}\|b\|_{C \dot{M} O^{s_{i}\left(\mathbb{R}^{n}\right)}}\right|^{q} d x\right)^{1 / q} \\
& \leq C \prod_{i=1}^{m}\|b\|_{C \dot{M} O^{s_{i}\left(\mathbb{R}^{n}\right)}}\left(r^{(\beta-n / p) q} \int_{B(0, r)} d x\right)^{1 / q} \\
& \leq C \prod_{i=1}^{m}\|b\|_{C \dot{M} O^{s_{i}}\left(\mathbb{R}^{n}\right)}
\end{aligned}
$$

For $K_{21}$,

$$
\begin{aligned}
& \left(\int_{B(0, r)} \mid \frac{1}{|x|^{n-\beta}} \prod_{i \in \sigma}\left(b_{i}(x)-\lambda_{i}\right)\right. \\
& \left.\times\left.\int_{|y|<|x|} \tilde{a}(y) \prod_{i \in \sigma^{c}}\left(\lambda_{i}-b_{i}(y)\right) d y\right|^{q} d x\right)^{1 / q} \\
& \leq C\left(\int_{B(0, r)} \mid \frac{1}{r^{n / p-\beta}}\right. \\
& \left.\times\left.\prod_{i \in \sigma}\left(b_{i}(x)-\lambda_{i}\right) \prod_{i \in \sigma^{c}}\|b\|_{C \dot{M} O^{s_{i}}\left(\mathbb{R}^{n}\right)}\right|^{q} d x\right)^{1 / q} \\
& \leq C \prod_{i \in \sigma^{c}}\|b\|_{C \dot{M} O^{s_{i}\left(\mathbb{R}^{n}\right)}}\left(r^{-n} \int_{B(0, r)}\left|\prod_{i \in \sigma}\left(b_{i}(x)-\lambda_{i}\right)\right|^{q} d x\right)^{1 / q} \\
& \leq C \prod_{i \in \sigma^{c}}\|b\|_{C \dot{M} O^{s_{i}}\left(\mathbb{R}^{n}\right)} \cdot \prod_{i \in \sigma}\|b\|_{C \dot{M} O^{s_{i} q}\left(\mathbb{R}^{n}\right)} \\
& \leq C \prod_{i=1}^{m}\|b\|_{C \dot{M} O^{\max \left\{s_{i} q, s_{i}\right\}}\left(\mathbb{R}^{n}\right)} .
\end{aligned}
$$

For $x \in \mathbb{R}^{n} \backslash B(0, r)$, we have $|x| \geq r$; then $\{y:|y|<|x|\} \bigcap\{y$ : $|y|<r\}=\{y:|y|<r\}$. By the condition of $\int_{B} a(y) d y=$ $\int_{B} a(y) \prod_{j \in \sigma} b_{j} d y=0$ for any $\sigma \in C_{i}^{m}, 1 \leq j \leq m$, we have that

$$
\begin{aligned}
& \int_{|y|<r} \tilde{a}(y) \prod_{i \in \sigma^{c}}\left(\lambda_{i}-b_{i}(y)\right) d y \\
& =\int_{|y|<r} \tilde{a}(y) \prod_{i=1}^{m}\left(\lambda_{i}-b_{i}(y)\right) d y=0,
\end{aligned}
$$

so,

$$
K_{12}=K_{22}=K_{32}=0
$$

Combining all the above estimates, we complete the proof of Theorem 32 . 


\section{Conflict of Interests}

The authors declare that they do not have any commercial or associative interests that represents a conflict of interests in connection with the work submitted.

\section{Acknowledgments}

Jiang Zhou is supported by the National Science Foundation of China (Grant nos. 11261055 and 11161044) and the National Natural Science Foundation of Xinjiang (Grant nos. 2011211A005 and BS120104).

\section{References}

[1] G. Hardy, J. Littlewood, and G. Polya, Inequalities, Cambridge University Press, New York, NY, USA, 1934.

[2] K. Andersen and B. Muckenhoupt, "Weighted weak type Hardy inequalities with applications to Hilbert transforms and maximal functions," Studia Mathematica, vol. 72, no. 1, pp. 9-26, 1982.

[3] M. Christ and L. Grafakos, "Best constants for two nonconvolution inequalities," Proceedings of the American Mathematical Society, vol. 123, no. 6, pp. 1687-1693, 1995.

[4] E. Sawyer, "Weighted Lebesgue and Lorentz norm inequalities for the Hardy operator," Transactions of the American Mathematical Society, vol. 281, no. 1, pp. 329-337, 1984.

[5] Z.-W. Fu, Z.-G. Liu, S.-Z. Lu, and H.-B. Wang, "Characterization for commutators of $n$-dimensional fractional Hardy operators," Science in China A, vol. 50, no. 10, pp. 1418-1426, 2007.

[6] B. I. Golubov, "On the boundedness of the Hardy and the Hardy-Littlewood operators in the spaces $\mathrm{Re}^{1}$ and BMO," Sbornik Mathematics, vol. 188, no. 7, pp. 1041-1054, 1997.

[7] S. C. Long and J. Wang, "Commutators of Hardy operators," Journal of Mathematical Analysis and Applications, vol. 274, no. 2, pp. 626-644, 2002.

[8] Y. Komori, "Notes on commutators of Hardy operators," International Journal of Pure and Applied Mathematics, vol. 7, no. 3, pp. 329-334, 2003.

[9] S. Z. Lu and D. C. Yang, "The central BMO spaces and Littlewood-Paley operators," Approximation Theory and Its Applications, vol. 11, no. 3, pp. 72-94, 1995.

[10] J. Xiao, " $L^{p}$ and BMO bounds of weighted Hardy-Littlewood averages," Journal of Mathematical Analysis and Applications, vol. 262, no. 2, pp. 660-666, 2001.

[11] F. Y. Zhao, Z. W. Fu, and S. Z. Lu, "Endpoint estimates for $n$ dimensional Hardy operators and their commutators," Science China Mathematics, vol. 55, no. 10, pp. 1977-1990, 2012.

[12] Z. W. Fu, S. Z. Lu, and F. Y. Zhao, "Commutators of $n$-dimensional rough Hardy operators," Science China Mathematics, vol. 54, no. 1, pp. 95-104, 2011.

[13] S. Z. Lu, D. Y. Yan, and F. Y. Zhao, "Sharp bounds for Hardy type operators on higher dimensional product spaces," Journal of Inequalities and Applications, vol. 2013, article 148, 11 pages, 2013.

[14] S. Z. Lu, Four Lectueres on Real $H^{p}$ Spaces, World Scientific, Singapore, 1995.

[15] E. M. Stein, Harmonic Analysis: Real-Variable Methods, Orthogonality, and Oscillatory Integrals, Princeton University Press, Princeton, NJ, USA, 1993.
[16] A. Torchinsky, Real-Variable Methods in Harmonic Analysis, vol. 123 of Pure and Applied Mathematics, Academic Press, New York, NY, USA, 1986.

[17] X. W. Li and D. C. Yang, "Boundedness of some sublinear operators on Herz spaces," Illinois Journal of Mathematics, vol. 40, no. 3, pp. 484-501, 1996.

[18] S. Z. Lu and D. C. Yang, "Oscillatory singular integrals on Hardy spaces associated with Herz spaces," Proceedings of the American Mathematical Society, vol. 123, no. 6, pp. 1695-1701, 1995.

[19] Z. W. Fu, Z. G. Liu, and S. Z. Lu, "Commutators of weighted Hardy operators on $\mathbb{R}^{n}$," Proceedings of the American Mathematical Society, vol. 137, no. 10, pp. 3319-3328, 2009.

[20] C. Pérez, "Endpoint estimates for commutators of singular integral operators," Journal of Functional Analysis, vol. 128, no. 1, pp. 163-185, 1995.

[21] X. Yu and S. Z. Lu, "Endpoint estimates for generalized commutators of Hardy operators on $H^{1}$ space," Journal of Function Spaces and Applications, vol. 2013, Article ID 410305, 11 pages, 2013.

[22] Q.-Y. Zheng and Z.-W. Fu, "Lipschitz estimates for commutators of $n$-dimensional fractional Hardy operators," Communications in Mathematical Research, vol. 25, no. 3, pp. 241-245, 2009.

[23] L. Grafakos and R. Torres, "Multilinear Calderón-Zygmund theory," Advances in Mathematics, vol. 165, no. 1, pp. 124-164, 2002.

[24] L. Grafakos and R. H. Torres, "On multilinear singular integrals of Calderón-Zygmund type," Publicacions Matemàtiques, vol. 46, no. 1, pp. 57-91, 2002.

[25] J. Zhou, Y.-H. Cao, and L. Li, "Estimates of commutators for multilinear operators on Herz-type spaces," Applied Mathematics, vol. 25, no. 2, pp. 177-184, 2010. 


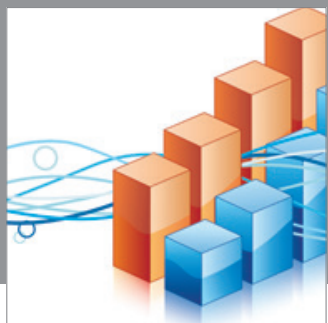

Advances in

Operations Research

mansans

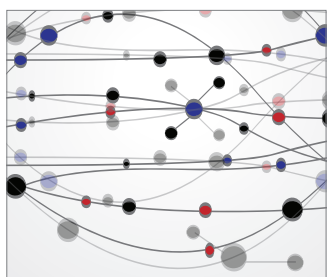

The Scientific World Journal
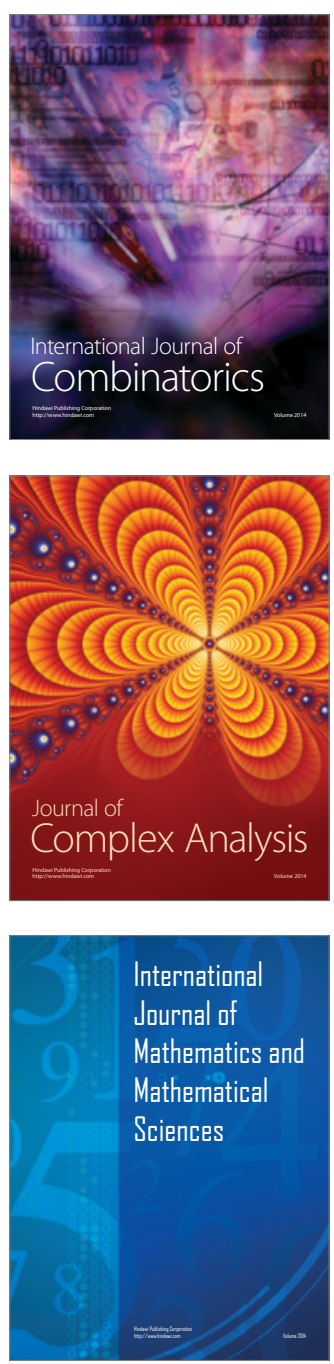
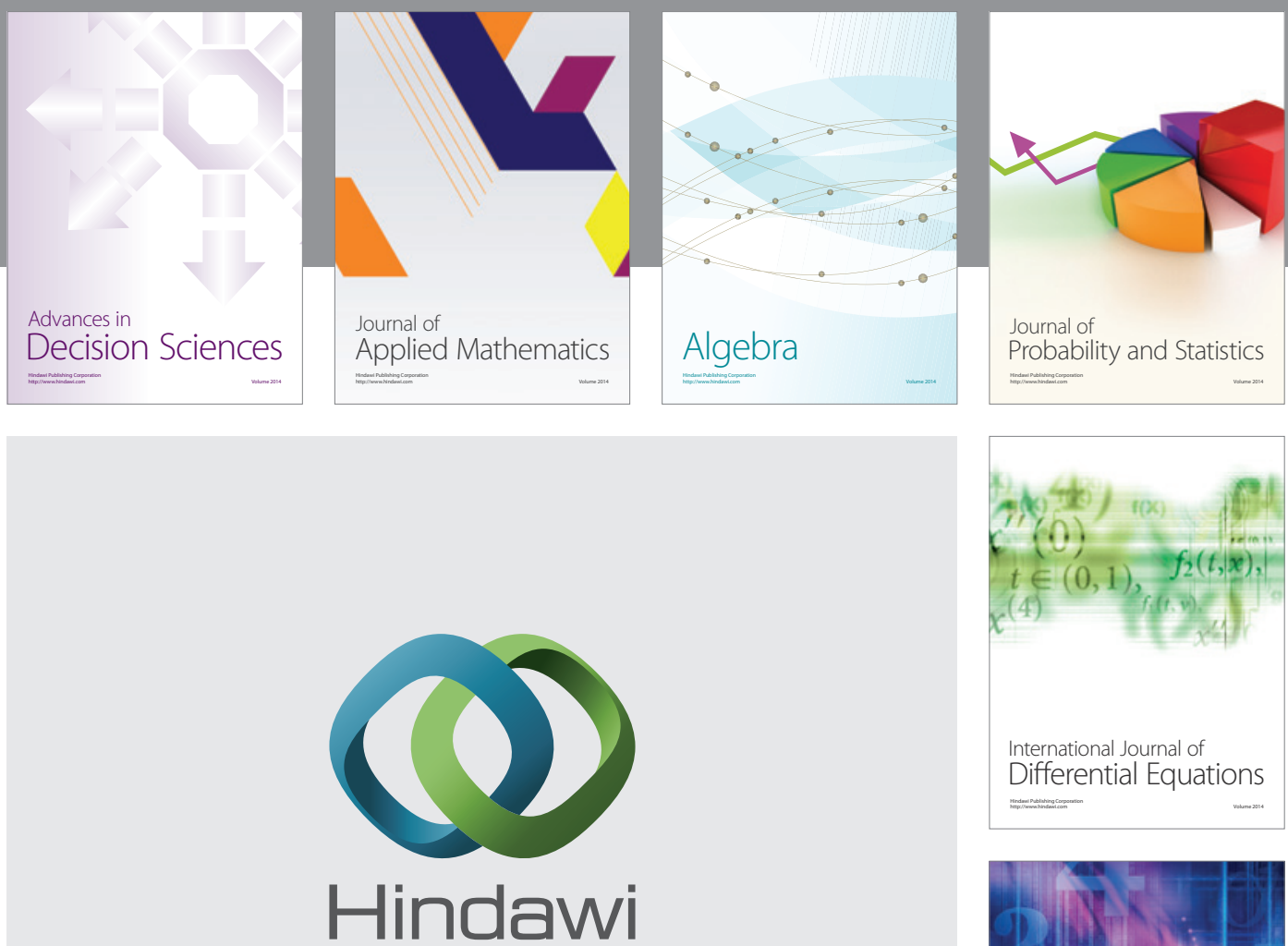

Submit your manuscripts at http://www.hindawi.com
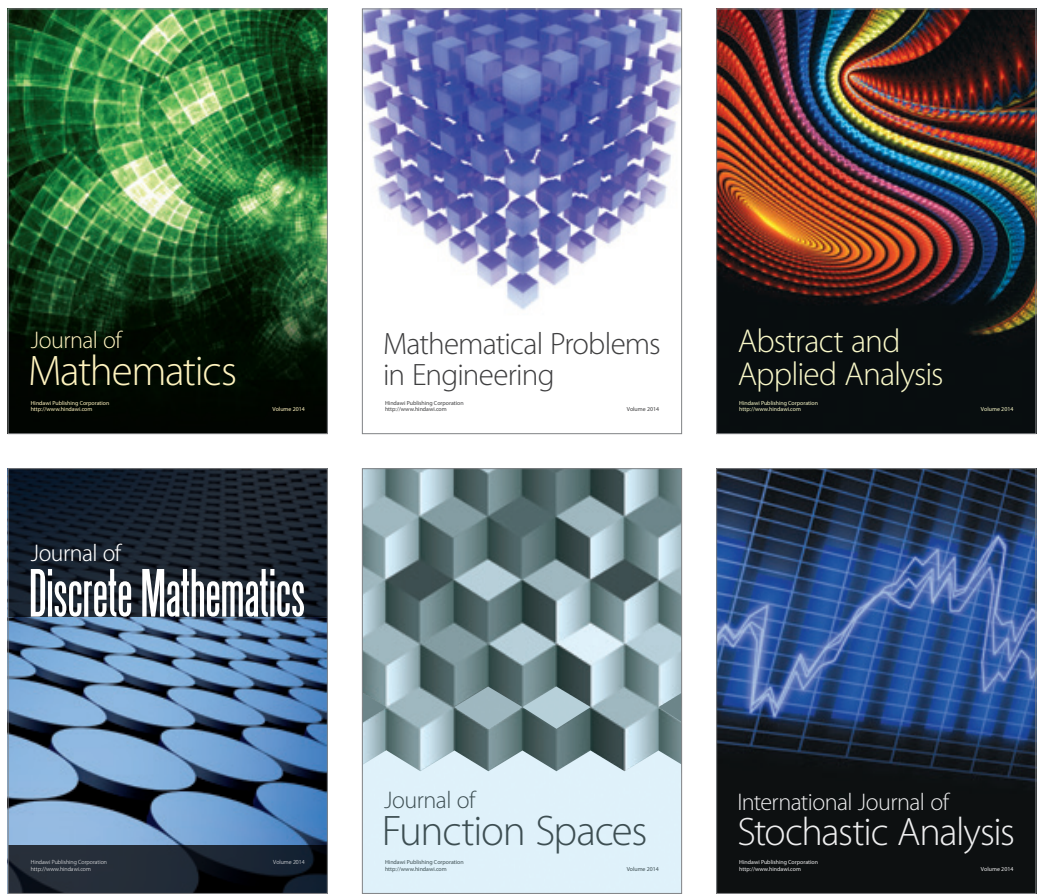

Journal of

Function Spaces

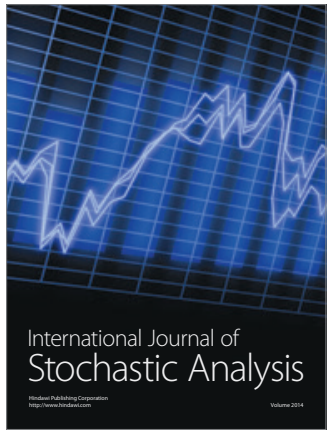

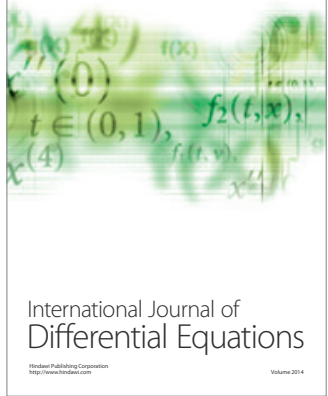
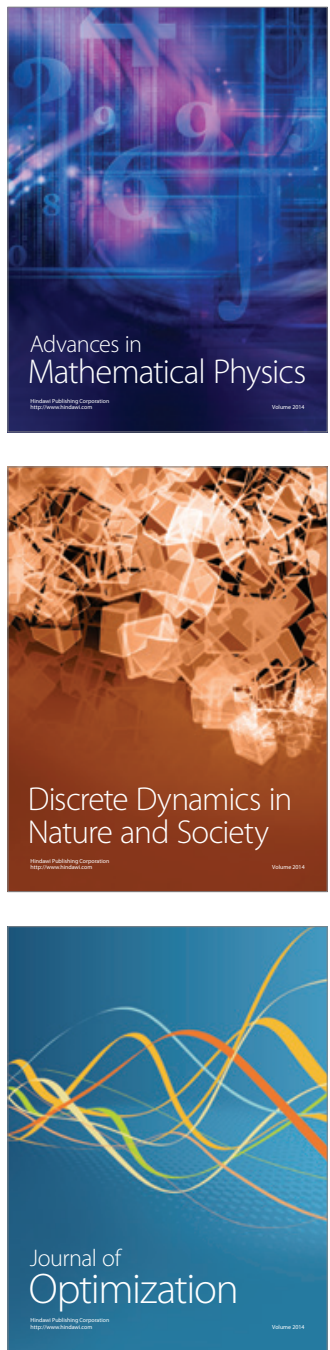